\title{
Associations of Perfluoroalkyl substances with blood lipids and Apolipoproteins in lipoprotein subspecies: the POUNDS-lost study
}

Gang Liư ${ }^{1,2}$, Bo Zhang ${ }^{3}$, Yang Hu², Jennifer Rood ${ }^{4}$, Liming Liang ${ }^{5}$, Lu Qi ${ }^{6}$, George A. Bray ${ }^{4}$, Lilian DeJonge? Brent Coull ${ }^{6,8}$, Philippe Grandjean ${ }^{9,10}$, Jeremy D. Furtado ${ }^{2}$ and Qi Sun ${ }^{2,11^{*}}$ (i)

\begin{abstract}
Background: The associations of perfluoroalkyl substance (PFAS) exposure with blood lipids and lipoproteins are inconsistent, and existing studies did not account for metabolic heterogeneity of lipoprotein subspecies. This study aimed to examine the associations between plasma PFAS concentrations and lipoprotein and apolipoprotein subspecies.

Methods: The study included 326 men and women from the 2-year Prevention of Obesity Using Novel Dietary Strategies (POUNDS) Lost randomized trial. Five PFASs, including perfluorooctanesulfonic acid (PFOS), perfluorooctanoic acid (PFOA), perfluorohexanesulfonic acid (PFHxS), perfluorononanoic acid (PFNA), and perfluorodecanoic acid (PFDA), were measured in plasma at baseline. For lipoprotein and apolipoprotein subspecies, total plasma was fractionated first by apolipoprotein (apo) C-III content and then by density. Each subfraction was then measured for apoB, apoC-III, and apoE concentrations, as well as triglyceride and cholesterol contents, both at baseline and at 2 years.

Results: For lipids and apolipoproteins in total plasma at baseline, elevated plasma PFAS concentrations were significantly associated with higher apoB and apoC-III concentrations, but not with total cholesterol or triglycerides. After multivariate adjustment of lifestyle factors, lipid-lowering medication use, and dietary intervention groups, PFAS concentrations were primarily associated with lipids or apolipoprotein concentrations in intermediate-to-low density lipoprotein $(I D L+L D L)$ and high-density lipoprotein $(H D L)$ that contain apoC-III. Comparing the highest and lowest tertiles of PFOA, the least-square means (SE) (mg/dl) were $4.16(0.4)$ vs $3.47(0.4)$ for apoB ( $P$ trend $=$ $0.04), 2.03(0.2)$ vs $1.66(0.2)$ for apoC-III ( $P$ trend $=0.04)$, and $8.4(0.8)$ vs $6.8(0.8)$ for triglycerides $(P$ trend $=0.03)$ in $I D L+L D L$ fraction that contains apoC-III. For HDL that contains apoC-III, comparing the highest and lowest tertiles of PFOA, the least-square means (SE) $(\mathrm{mg} / \mathrm{dl})$ of apoC-III were $11.9(0.7)$ vs $10.4(0.7)(P$ trend $=0.01)$. In addition, elevated PFNA and PFDA concentrations were also significantly associated with higher concentrations of apoE in HDL that contains apoC-III ( $P$ trend $<0.01$ ). Similar patterns of associations were demonstrated between baseline PFAS concentrations and lipoprotein subspecies measured at 2 years. Baseline PFAS levels were not associated with changes in lipoprotein subspecies during the intervention.
\end{abstract}

(Continued on next page)

\footnotetext{
* Correspondence: qisun@hsph.harvard.edu

${ }^{2}$ Department of Nutrition, Harvard T.H. Chan School of Public Health, 665 Huntington Ave, Boston, MA 02115, USA

${ }^{11}$ Channing Division of Network Medicine, Department of Medicine, Brigham and Women's Hospital and Harvard Medical School, Boston, MA, USA

Full list of author information is available at the end of the article
}

(c) The Author(s). 2020 Open Access This article is distributed under the terms of the Creative Commons Attribution 4.0 International License (http://creativecommons.org/licenses/by/4.0/), which permits unrestricted use, distribution, and reproduction in any medium, provided you give appropriate credit to the original author(s) and the source, provide a link to the Creative Commons license, and indicate if changes were made. The Creative Commons Public Domain Dedication waiver (http://creativecommons.org/publicdomain/zero/1.0/) applies to the data made available in this article, unless otherwise stated. 
(Continued from previous page)

Conclusions: Our results suggest that plasma PFAS concentrations are primarily associated with blood lipids and apolipoproteins in subspecies of IDL, LDL, and HDL that contain apoC-III, which are associated with elevated cardiovascular risk in epidemiological studies. Future studies of PFAS-associated cardiovascular risk should focus on lipid subfractions.

Keywords: Perfluoroalkyl substance, Lipid subfractions, Epidemiology

\section{Introduction}

Per- and polyfluoroalkyl substances (PFASs) are extensively used in many industrial and consumer products including stain- and water-repellent fabrics, nonstick cookware, and food packing [1]. PFASs have structural homology with fatty acids and may interfere with lipid metabolism [2], probably through activating peroxisome proliferator-activated receptors (PPAR) [3].

Most animal studies reported decreases in lipids after high-dose administration of perfluorooctanoic acid (PFOA) and perfluorooctane sulfonate (PFOS) [1], although some human studies have shown increased blood lipids at higher PFAS exposures [1, 4-7], especially when this association was examined prospectively among individuals exposed to high levels of PFOA [8, 9]. However, this positive association has not been uniformly replicated in other populations at lower exposure levels $[10,11]$, and data from occupational exposures are in dispute [12].

Potential reasons for these inconsistent findings may involve differences in study designs and exposure levels, and another important concern is the metabolic and functional heterogeneity of lipoprotein subspecies [13, 14]. Thus, what is commonly referred to as low-density lipoprotein (LDL), high-density lipoprotein (HDL), and other lipid fractions constitute diverse groups of lipoprotein particles with heterogeneous biological functions, varying in cholesterol and triglyceride contents, as well as attachments of apolipoprotein (apo) $\mathrm{C}$ and apoE [13, 15-17]. Accumulating evidence has suggested that apoC-III acts as an independent risk factor for cardiovascular disease [18-20]. Further, the apoC-III attached to HDL could significantly attenuate the beneficial metabolic action of HDL apoE and subsequently affect lipid metabolism and cardiovascular disease risk [17]. So far, association between PFAS exposures and lipoprotein subspecies has apparently not been investigated.

To fill this knowledge gap, we examined the associations of plasma PFAS concentrations with lipoproteins and apolipoprotein subspecies in men and women participating in the Prevention of Obesity Using Novel Dietary Strategies (POUNDS) Lost trial. We specifically hypothesized that PFAS concentrations would be positively associated with lipoprotein subspecies that contain apoC-III.

\section{Methods \\ Study population}

The POUNDS Lost study (ClinicalTrials.gov number: NCT00072995) was a randomized clinical trial designed to compare the effects of four energy-reduced diets with different compositions of macronutrients (i.e., fat, protein, and carbohydrate) on weight loss. The trial was conducted at two sites: the Harvard T.H. Chan School of Public Health and Brigham and Women's Hospital, Boston; and the Pennington Biomedical Research Center of the Louisiana State University System, Baton Rouge, from October 2004 through December 2007. The details have been described previously [21]. Briefly, 811 overweight and obese men and women aged 30-70 years were randomly assigned to one of four healthy diets that followed the American Heart Association recommendations for cardiovascular health at baseline, and 645 participants (80\%) completed the trial at 2 years [21]. The main finding of this trial was that weight changes were not significantly different between diet groups [21]. Of these participants, 406 were randomly selected for analysis of lipoprotein subspecies at baseline and 2 years. The current analysis finally included 326 participants who had data on both PFASs and lipoprotein subspecies. The protocol was approved by the Institutional Review Board at Harvard T.H. Chan School of Public Health, Brigham and Women's Hospital, and the Pennington Biomedical Research Center of the Louisiana State University System, as well as by a data and safety monitoring board appointed by the National Heart, Lung, and Blood Institute. All participants provided written informed consent.

\section{Laboratory measurements of PFAS}

Plasma concentrations of PFOS, PFOA, perfluorohexanesulfonic acid (PFHxS), perfluorononanoic acid (PFNA), and perfluorodecanoic acid (PFDA) were measured by a sensitive and reliable method based on online solid phase extraction and liquid chromatography coupled to a triple quadrupole mass spectrometer [22], with minor modifications. The concentrations of the five PFASs were all above the limit of detection $(0.05 \mathrm{ng} /$ $\mathrm{mL}$ ), and the inter- and intra-assay coefficients of variation $(\mathrm{CV})$ were both $<10 \%$. PFAS concentrations in 
our study population were comparable to concentrations in the general US population in 2003-2004 [23].

\section{Laboratory measurements of lipoprotein and lipoprotein subspecies}

The methodology of lipoprotein subspecies quantification has been described previously [24]. Briefly, plasma was thawed and incubated overnight at $4{ }^{\circ} \mathrm{C}$ in antiapoC-III immuno-affinity columns to bind lipoproteins containing apoC-III. The unbound plasma fraction (CIII-) was eluted with phosphate-buffered saline and the bound lipoproteins (CIII+) were eluted with $3 \mathrm{M}$ sodium thiocyanate. Very-low density lipoprotein (VLDL) was isolated from each fraction by ultracentrifugation at $4{ }^{\circ} \mathrm{C}$ and $25,000 \mathrm{rpm}$ for $16 \mathrm{~h}$. The combined intermediate-density lipoprotein (IDL) and low-density lipoprotein (LDL) fraction was then isolated following density adjustment with potassium bromide to $\mathrm{d}=1.063$ $\mathrm{g} / \mathrm{mL}$ by ultracentrifugation at $4{ }^{\circ} \mathrm{C}$ and $25,000 \mathrm{rpm}$ for $24 \mathrm{~h}$. The remaining solution contained the HDL and other components of plasma. Therefore, six lipoprotein subspecies were generated: VLDL that contains or lacks apoC-III, IDL + LDL that contains or lacks apoC-III, and HDL that contains or lacks apoC-III. Among these subspecies, apoB, apoC-III, and apoE concentrations were further assayed using sandwich ELISAs (Academy Biomedical, Houston, TX), and cholesterol and triglyceride concentrations were determined using enzymatic assays (Thermo Electron Corp, Waltham, MA). The samples from the same participants were assayed in the same run by the same technicians in a random sequence, and any sample with an intra-assay $\mathrm{CV}>$ $15 \%$ was repeated.

\section{Assessments of covariates}

Using standardized questionnaires, we obtained information on age, sex, race, educational attainment, smoking status, and alcohol consumption [25]. Physical activity was estimated using the Baecke physical activity questionnaire, which included 16 items inquiring about levels of habitual physical activities [26]. Body weight and waist circumference were measured at baseline, 6, 12, 18, and 24 months. Body mass index (BMI) was calculated as body weight in kilograms divided by height in meters squared.

\section{Statistical methods}

The comparisons between participants included in the current analysis and those excluded were tested by the Student's $t$ test for normally distributed variables, the Wilcoxon rank-sum test for skewed variables, and the chisquare test for categorical variables. The associations between baseline PFAS and the lipoprotein and lipoprotein subspecies at baseline and at 2 years after intervention were examined using linear regression models. The leastsquare means and standard error (SE) of lipoprotein and lipoprotein subspecies according to tertiles of PFAS concentrations were calculated.

In terms of multivariate adjustment, we considered traditional covariates, including demographic, socioeconomic, and lifestyle factors. In addition, given the clinical trial study design, we further included dietary intervention groups in the model. Lastly, considering that lipid-lowering medication use might confounder the association of interest, we also took this variable into account in multivariate analyses. Specifically, covariates considered in the multivariate models included age (years), sex (men, women), race (white, non-white), educational attainment (high school or less, some college, and college graduate or beyond), smoking status (never, former, and current smoker), alcohol consumption (drinks/week), physical activity (MET-hr/wk), BMI (kg/ $\mathrm{m}^{2}$ ), four dietary intervention groups (categorical), and regular lipid-lowering medication use (yes or no). Tests of linear trend across increasing tertiles of PFAS were examined by assigning a median value to each tertile and treating it as a continuous variable. In addition, we modeled log-transformed (base 10) PFAS concentration as continuous variables.

Several sensitivity analyses were performed. First, the associations of PFAS with lipoprotein and lipoprotein subspecies were examined in strata defined by sex and race, and partial Spearman correlation coefficients $\left(r_{s}\right)$ were calculated to evaluate strength of associations. Among women, we further adjusted for menopausal status (yes or no) and hormone replacement therapy use (yes or no). Second, analyses were further restricted to participants without lipid-lowering medication use or to non-current smokers. A two-sided $P<0.05$ was considered statistically significant. These statistical analyses were performed with SAS software, version 9.4 (SAS Institute Inc., Cary, North Carolina).

\section{Results}

The baseline characteristics of participants included in the current study $(n=326)$ are shown in Table 1 . The mean (SD) age of the participants was 52.7 (8.7) years, with a mean (SD) BMI of $32.3(3.8) \mathrm{kg} / \mathrm{m}^{2}$. For IDL + LDL and HDL, the concentrations of these species that contain apoC-III were much lower than their counterparts that lack apoC-III. Additional file 1: Figure S1 shows a heatmap of correlations between lipoprotein and apolipoprotein subspecies. There were moderate to high correlations among most of the lipoprotein and apolipoprotein subspecies $\left(r_{s}\right.$ ranged from -0.29 to 0.97 , $P<0.001)$. Significant inter-correlations were observed between PFOS, PFOA, PFHxS, PFNA, and PFDA $\left(r_{\mathrm{s}}\right.$ between 0.32 and 0.84 ). In addition, in comparison with 
Table 1 Baseline characteristics of participants in the POUNDS Lost study

\begin{tabular}{ll}
\hline $\mathrm{N}$ & 326 \\
\hline Age (years) & $52.7 \pm 8.7$ \\
Sex, men, \% & 39.0 \\
Race, \% & \\
$\quad$ White & 85.3 \\
$\quad$ Black & 11.0 \\
$\quad$ Hispanic & 2.8 \\
$\quad$ Other & 0.9 \\
BMl (kg/m²) & $32.3 \pm 3.8$ \\
Weight (kg) & $91.7 \pm 15.3$ \\
Weight (lbs) & $202.2 \pm 33.8$ \\
Waist circumference (cm) & $103.2 \pm 12.9$ \\
Education level, high school or less, \% & 9.8 \\
Current smoker, yes, \% & 3.4 \\
Alcohol consumption (drinks/week) & $2.1 \pm 2.8$ \\
Physical activity ${ }^{a}$ & $1.6 \pm 0.1$ \\
Systolic blood pressure (mmHg) & $119.8 \pm 13.1$ \\
Diastolic blood pressure (mmHg) & $75.1 \pm 8.9$ \\
Glucose (mg/dl) & $92.1 \pm 11.3$ \\
PFOS (ng/ml) & $23.5(15.9,38.0)$ \\
PFOA (ng/ml) & $4.6(3.3,6.4)$ \\
PFHxS (ng/ml) & $2.4(1.6,3.6)$ \\
PFNA (ng/ml) & $1.5(1.0,2.3)$ \\
Total plasma (mg/dl) & $0.4(0.2,0.5)$ \\
& \\
\hline &
\end{tabular}

$\begin{array}{ll}\text { Total cholesterol } & 188.9(168.0,225.3) \\ \text { Triglycerides } & 96.9(69.3,140.6) \\ \text { ApoB } & 85.8(69.3,110.5) \\ \text { ApoE } & 7.7(6.1,9.3) \\ \text { ApoC-III } & 13.4(10.6,17.1) \\ \text { IDL + LDL containing apoC-III (mg/dl) } & \\ \text { Cholesterol } & 7.2(5.2,10.2) \\ \text { Triglycerides } & 5.7(3.9,8.3) \\ \text { ApoB } & 2.8(1.8,4.5) \\ \text { ApoE } & 0.20(0.13,0.30) \\ \text { ApoC-III } & 1.4(0.9,2.1) \\ \text { IDL + LDL lacking apoC-III (mg/dl) } & \\ \text { Cholesterol } & 107.0(86.9,129.5) \\ \text { Triglycerides } & 18.2(13.2,27.5) \\ \text { ApoB } & 72.7(58.0,92.8) \\ \text { ApoE } & 0.50(0.35,0.76) \\ \text { HDL containing apoC-III (mg/dl) } & \\ \text { Cholesterol } & 6.1(4.7,8.2) \\ \text { Triglycerides } & 2.4(1.6,3.7) \\ & \end{array}$

Table 1 Baseline characteristics of participants in the POUNDS Lost study (Continued)

\begin{tabular}{ll}
\hline $\mathrm{N}$ & 326 \\
\hline ApoE & $4.1(3.1,4.8)$ \\
$\quad$ ApoC-III & $10.7(8.0,13.9)$ \\
HDL lacking apoC-III (mg/dl) & \\
Cholesterol & $54.9(45.7,67.4)$ \\
Triglycerides & $6.3(4.6,9.6)$ \\
ApoE & $2.2(1.5,3.2)$ \\
\hline
\end{tabular}

Data are mean $\pm S D$, median (interquartile range), or percentage (\%). ${ }^{\text {a Physical }}$ activity was estimated by the Baecke Questionnaire. BMI Body mass index, TG Triglycerides, PFOS Perfluorooctane sulfonate, PFOA Perfluorooctanoate, PFHXS Perfluorohexanesulfonate, PFNA Perfluorononanoic acid, PFDA Perfluorodecanoic acid

the remaining participants not included in the current study, the participants included were slightly older (52.7 vs 49.6 years, $P<0.001$ ) and more likely to be white (85.3\% vs $75.3 \%, P=0.01)$. There was otherwise no significant difference in their characteristics (Additional file 1: Table S1).

After multivariate adjustment including demographic and lifestyle factors, BMI, and dietary intervention groups, baseline PFOA concentrations were positively associated with apoB and apoC-III concentrations in plasma (Table 2). Comparing the highest vs lowest tertiles, the least-square means $(\mathrm{SE})(\mathrm{mg} / \mathrm{dl})$ were 95.4 (5.1) and $85.7(5.3)$ for apoB ( $P$ trend $=0.03)$ and 15.5 $(0.9)$ and $13.4(0.9)$ for apoC-III $(P$ trend $=0.007)$. Similar results were observed when PFOA concentrations were treated as continuous variables; for each unit increment of $\log _{10}$-transformed PFOA, there was an increment of $0.11 \mathrm{mg} / \mathrm{dl}$ in $\log _{10}$-transformed apoB $(P=$ $0.007)$ and an increment of $0.15 \mathrm{mg} / \mathrm{dl}$ in $\log _{10}$-transformed apoC-III $(P=0.003)$ (Table 2$)$. PFASs were not associated with plasma concentrations of total triglycerides or cholesterol.

Figure 1 shows the associations between PFASs and lipid and apolipoprotein subspecies in IDL+ LDL that contains or lacks apoC-III. After multivariate adjustment, PFAS were primarily associated with lipids/apolipoproteins in IDL and LDL fraction that contains apoCIII. Comparing the highest and lowest tertiles of PFOA, the least-square means $(\mathrm{SE})(\mathrm{mg} / \mathrm{dl})$ were $4.16(0.4)$ vs $3.47(0.4)$ for apoB ( $P$ trend $=0.04), 2.03(0.2)$ vs 1.66 $(0.2)$ for apoC-III ( $P$ trend $=0.04$ ), and $8.4(0.8)$ vs 6.8 $(0.8)$ for triglycerides $(P$ trend $=0.03)$ in IDL and LDL fraction that contains apoCIII (Fig. 1). Significant results were also observed when PFOA concentrations were treated as continuous variables; for each unit increment of $\log _{10}$-transformed PFOA, there was a $0.15 \mathrm{mg} / \mathrm{dl}$ increment in triglycerides $(P=0.03), 0.22 \mathrm{mg} / \mathrm{dl}$ increment of apoB $(P=0.01)$, and $0.24 \mathrm{mg} / \mathrm{dl}$ increment in apoC-III $(P=0.007)$. In addition, we also found some 
Table 2 Least-square means of baseline lipids and apolipoproteins in total plasma according to tertile of PFAS

\begin{tabular}{|c|c|c|c|c|c|}
\hline & Total Cholesterol & Triglycerides & ApoB & ApoE & ApoC-III \\
\hline \multicolumn{6}{|l|}{ PFOS (ng/ml) } \\
\hline $\mathrm{T} 1(<18.8)$ & $180.9 \pm 8.0$ & $126.8 \pm 11.6$ & $86.6 \pm 5.4$ & $7.9 \pm 0.4$ & $13.7 \pm 0.9$ \\
\hline T2 (18.8-33.1) & $189.3 \pm 7.9$ & $132.4 \pm 11.4$ & $90.3 \pm 5.3$ & $8.2 \pm 0.4$ & $15.2 \pm 0.9$ \\
\hline T3 (> 33.1) & $190.7 \pm 7.3$ & $126.1 \pm 10.5$ & $94.0 \pm 4.9$ & $8.4 \pm 0.4$ & $14.8 \pm 0.8$ \\
\hline$P_{\text {trend }}$ & 0.21 & 0.80 & 0.11 & 0.22 & 0.36 \\
\hline \multicolumn{6}{|l|}{ PFOA (ng/ml) } \\
\hline $\mathrm{T} 1(<3.7)$ & $189.1 \pm 7.9$ & $111.1 \pm 11.2$ & $85.7 \pm 5.3$ & $7.9 \pm 0.4$ & $13.4 \pm 0.9$ \\
\hline T2 (3.7-5.6) & $189.3 \pm 7.6$ & $137.3 \pm 10.8$ & $91.2 \pm 5.0$ & $8.2 \pm 0.4$ & $14.7 \pm 0.9$ \\
\hline T3 (> 5.6) & $188.4 \pm 7.7$ & $131.8 \pm 10.9$ & $95.4 \pm 5.1$ & $8.4 \pm 0.4$ & $15.5 \pm 0.9$ \\
\hline$P_{\text {trend }}$ & 0.67 & 0.06 & 0.03 & 0.12 & 0.007 \\
\hline \multicolumn{6}{|l|}{ PFHxS (ng/ml) } \\
\hline $\mathrm{T} 1(<1.8)$ & $181.6 \pm 7.8$ & $119.4 \pm 11.2$ & $90.7 \pm 5.2$ & $8.4 \pm 0.4$ & $13.9 \pm 0.9$ \\
\hline T2 (1.8-3.1) & $189.3 \pm 7.6$ & $133.6 \pm 11.0$ & $89.7 \pm 5.1$ & $8.2 \pm 0.4$ & $14.9 \pm 0.9$ \\
\hline T3 (> 3.1) & $192.5 \pm 7.8$ & $130.8 \pm 11.2$ & $93.0 \pm 5.2$ & $8.1 \pm 0.4$ & $15.0 \pm 0.9$ \\
\hline$P_{\text {trend }}$ & 0.15 & 0.37 & 0.55 & 0.39 & 0.28 \\
\hline \multicolumn{6}{|l|}{ PFNA (ng/ml) } \\
\hline $\mathrm{T} 1(<1.1)$ & $185.6 \pm 7.7$ & $132.9 \pm 11.1$ & $87.6 \pm 5.2$ & $7.9 \pm 0.4$ & $14.4 \pm 0.9$ \\
\hline T2 (1.1-1.8) & $186.1 \pm 7.8$ & $128.2 \pm 11.2$ & $89.8 \pm 5.2$ & $8.2 \pm 0.4$ & $14.4 \pm 0.9$ \\
\hline $\mathrm{T} 3$ (> 1.8) & $190.8 \pm 7.5$ & $123.6 \pm 10.8$ & $95.0 \pm 5.0$ & $8.4 \pm 0.4$ & $15.0 \pm 0.9$ \\
\hline$P$ trend & 0.39 & 0.35 & 0.08 & 0.20 & 0.38 \\
\hline \multicolumn{6}{|l|}{ PFDA (ng/ml) } \\
\hline $\mathrm{T} 1(<0.3)$ & $183.1 \pm 7.9$ & $138.9 \pm 11.3$ & $89.0 \pm 5.3$ & $7.9 \pm 0.4$ & $14.5 \pm 0.9$ \\
\hline T2 (0.3-0.5) & $186.6 \pm 7.5$ & $119.7 \pm 10.7$ & $88.2 \pm 5.0$ & $8.1 \pm 0.4$ & $14.3 \pm 0.9$ \\
\hline $\mathrm{T} 3(>0.5)$ & $192.1 \pm 7.6$ & $129.3 \pm 10.8$ & $95.6 \pm 5.0$ & $8.5 \pm 0.4$ & $15.0 \pm 0.9$ \\
\hline$P_{\text {trend }}$ & 0.17 & 0.32 & 0.13 & 0.12 & 0.52 \\
\hline
\end{tabular}

Data are least squares (LS) means \pm standard error (SE). ${ }^{2}$ Values were adjusted for age (years), sex (men, women), race (White, non-White), educational attainment (high school or less, some college, and college graduate or beyond), smoking status (never, former, and current smoker), alcohol consumption (drinks/week), physical activity (MET-hr/wk), BMI ( $\mathrm{kg} / \mathrm{m}^{2}$ ), regular lipid-lowering medication use (yes or no), and dietary intervention groups (categorical)

positive associations for other PFASs. Comparing the highest vs lowest tertiles, the least-square means (SE) $(\mathrm{mg} / \mathrm{dl})$ of triglycerides were $8.5(0.8)$ vs $6.6(0.8)$ for PFHxS $(P$ trend $=0.03)$, the least-square means $(\mathrm{SE})$ of cholesterol were $9.4(0.6)$ vs $7.8(0.7)$ for PFDA ( $P$ trend $=0.007)$. In contrast, no significant association was observed between PFASs and lipids and apolipoproteins in IDL and LDL fraction that lacks apoC-III (Fig. 1).

The associations between PFASs and lipids and apolipoproteins in HDL that contains or lacks apoC-III are shown in Fig. 2. Similar to findings regarding IDL and LDL subspecies, after multivariate adjustment, positive associations with PFASs were primarily observed in HDL that contains apoC-III. Comparing the highest vs lowest tertiles, the least-square means $(\mathrm{SE})(\mathrm{mg} / \mathrm{dl})$ of apoC-III were 11.9 $(0.7)$ vs $10.4(0.7)$ for PFOA ( $P$ trend $=0.01)$. Significant positive associations were also observed between both PFNA and PFDA and apoE, as well as between PFHxS and cholesterol concentrations. Comparing extreme tertiles, the least-square means $(\mathrm{SE})$ of apoE were 4.53 $(0.2)$ vs $3.91(0.2)$ for PFNA ( $P$ trend $=0.004)$ and 4.51 $(0.2)$ vs $3.96(0.2)$ for PFDA ( $P$ trend $=0.005)$, and these figures were $8.3(0.7)$ vs $6.5(0.7)$ for cholesterol concentrations by PFHxS tertiles $(P$ trend $=0.008)$. Consistent results were observed when PFAS concentrations were treated as continuous variables; for each unit increment of $\log _{10}$-transformed PFAS, there was a $0.14 \mathrm{mg} / \mathrm{dl}$ increment in apoC-III for PFOA $(P=0.009)$, a $0.11 \mathrm{mg} / \mathrm{dl}$ increment in apoE for PFNA $(P=0.002)$, and a $0.12 \mathrm{mg} / \mathrm{dl}$ increment in apoE for PFDA $(P=0.005)$ (Fig. 2). In contrast, a significant association was only observed between PFOA and triglycerides in HDL that lacks apoC-III (9.3 [0.8] vs $7.6[0.8], P<0.05)$.

The associations between PFASs and lipids and apolipoprotein subspecies in VLDL are shown in Additional file 1: Table S2. PFOA and PFOS were positively associated with apoB in VLDL that lacks apoC-III. In addition, PFOS concentrations were inversely associated with 


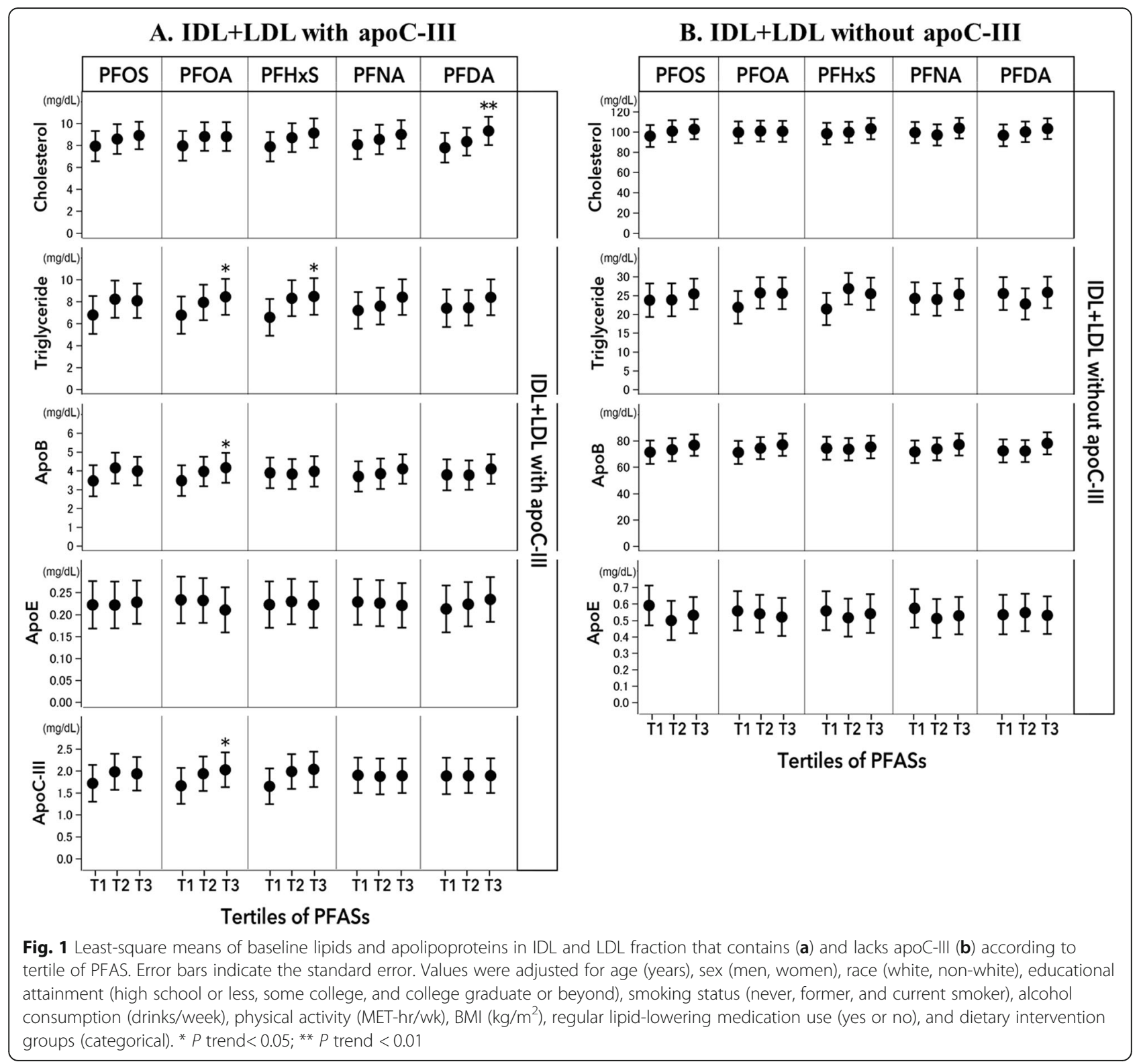

concentrations of apoE and cholesterol in VLDL that contains apoC-III, and PFNA was inversely associated with apoB, apoC-III, apoE, and cholesterol contents in VLDL that contains apoC-III.

Additional file 1: Table S3 shows the associations of PFASs with lipoprotein subspecies in men and women. In both genders, PFAS were positively associated with lipids or apolipoproteins in IDL + LDL and HDL that contain apoC-III, although stronger associations that contains apoC-III concentrations were observed in women. A similar pattern of associations was observed in race-stratified analyses, although most of the associations did not reach statistical significance among non-whites largely due to limited power ( $n=48$; data not shown). In addition, the results did not significantly change when menopausal status and hormone replacement therapy use were further adjusted, or when analysis was restricted to participants without lipid-lowering medication use or non-current smokers (data not shown). Similar patterns of associations were demonstrated between baseline PFAS concentrations and lipoprotein subspecies at 2 years after the intervention (Additional file 1: Table S4). PFASs were not associated with changes in lipoprotein subspecies from baseline to 2 years. No significant interaction was observed between dietary intervention groups and PFAS exposure on levels of lipoprotein subspecies.

\section{Discussion}

In this 2-year randomized trial in overweight and obese participants, we found that PFAS exposure levels were 


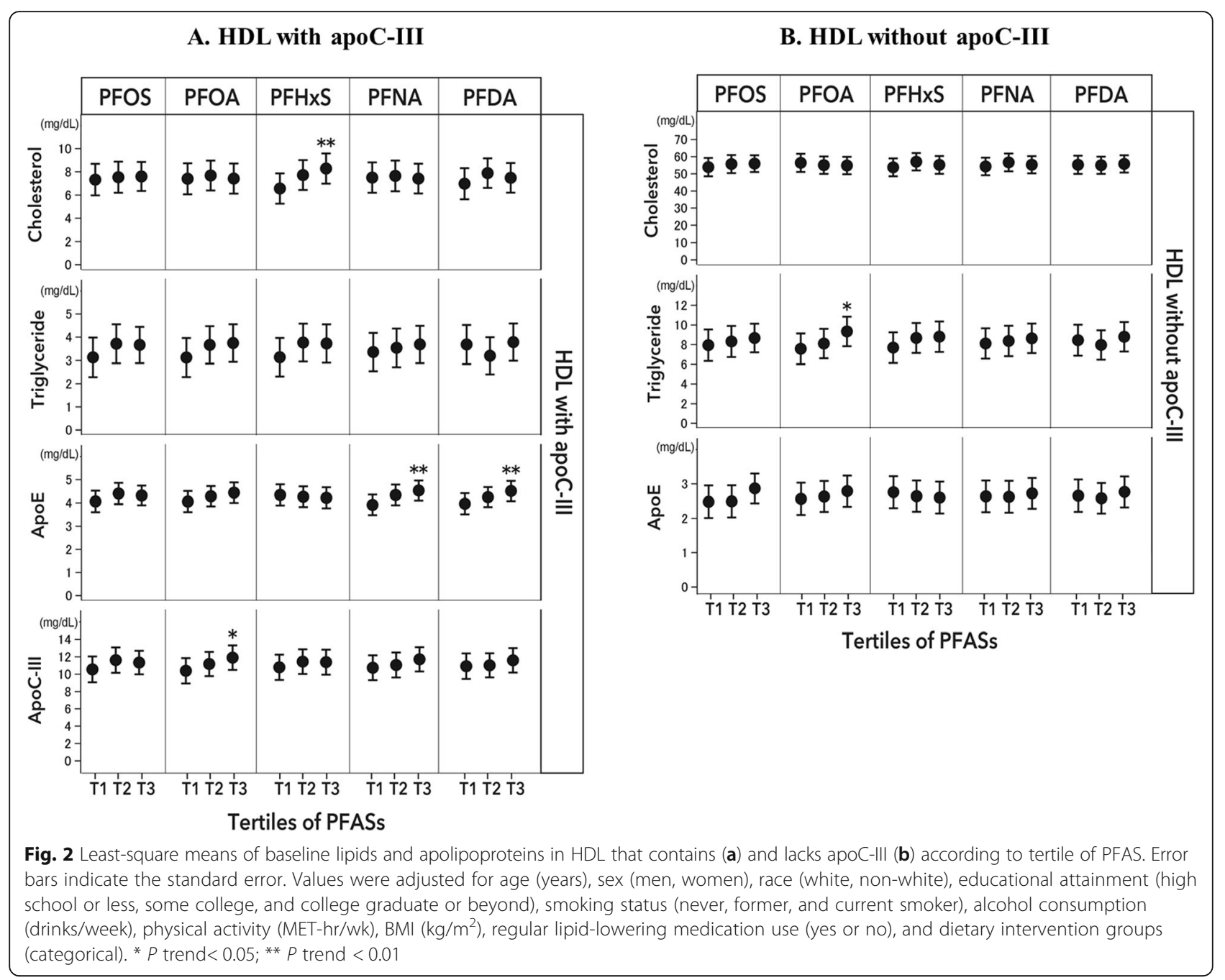

positively associated with plasma concentrations of apoB and apoC-III, but not with total cholesterol or triglycerides. Furthermore, higher PFAS concentrations were primarily associated with higher concentrations of cholesterol, triglycerides, and apolipoproteins in IDL, LDL, and HDL that contain apoC-III. These associations were independent of potential confounders including diet and lipid-lowering medication use and somewhat stronger among women. In addition, similar associations were observed when we examined baseline PFAS concentrations in relation to lipoprotein subspecies by the end of trial, thus suggesting that these findings were robust to changes in body weight during the trial.

Most animal studies showed that administration of PFAS led to lower levels of circulating lipids (i.e., total cholesterol and triglycerides), but known betweenspecies differences in PFAS toxicokinetics limit the generalizability of animal study findings to humans [1]. Evidence from human studies regarding PFAS exposure and lipids is mixed [11, 27, 28]. Positive associations between PFASs and total cholesterol, LDL cholesterol, or triglycerides were observed in some cross-sectional and prospective studies $[4,8,9,29-31]$, whereas other studies reported null associations or even inverse associations [11, 28, 32]. We are among the first to examine apolipoprotein species within the broad categories of lipoproteins that were not considered in previous studies $[13,14]$. Indeed, the present study showed no clear association between PFAS and total cholesterol, triglycerides, or lipoproteins in plasma, but PFAS exposures were associated with apoC-III levels and also the lipid contents in IDL, LDL, and HDL particles that contain apoCIII. The diverging functions of apolipoproteins in the same class of blood lipids may also explain the previous inconsistent findings regarding PFAS exposures and total blood lipid levels.

Current evidence suggests that blood lipid particles are highly heterogeneous and comprise a group of lipoproteins and apolipoproteins (such as apoC and apoE) with diverse biological functions [13, 15-17]. For instance, 
studies have shown that LDL that contains apoC-III, but not LDL that lacks apoC-III, was an independent risk factor for cardiovascular risk [18-20]. Two independent prospective studies showed that HDL cholesterol that contains or lacks apoC-III demonstrated opposite associations with the risk of coronary heart disease (CHD): HDL cholesterol that lacks apoC-III was inversely associated with $\mathrm{CHD}$, whereas HDL cholesterol that contains apoC-III (small subfraction) was associated with a higher risk of CHD [16]. Further, the associations of apoE concentrations in HDL with cardiovascular risk significantly differ in the presence of apoC-III in that HDL with both apoE and apoC-III tended to be associated with a higher cardiometabolic risk [17, 33, 34]. Therefore, the heterogeneous lipoprotein subspecies deserve to be characterized in order to improve the disease risk prediction rather than relying on total lipid fractions [14]. Our finding of PFAS exposures being primarily associated with IDL, LDL, and HDL subfractions containing apoC-III suggests that elevated PFAS exposure may potentially exert increased cardiovascular risk [19], as already suggested by recent evidence [35]. We estimated that the difference in apoC-III levels between the extreme tertiles of PFOA would lead to an $18 \%$ increased risk of cardiovascular disease (CVD), based on a pooled estimate of $148 \%$ increased CVD risk for each 5-mg/dl increase in apoC-III levels [20].

The mechanisms underlying our findings are not well understood. Evidence from animal studies has suggested that the biological effects of PFASs might be attributed to the activation of PPAR- $\alpha$ [3], but PFOA may also alter the expression of proteins regulated by hepatocyte nuclear factor $4 \alpha$ [36], a key regulator of lipid metabolism [37]. However, these findings may not be extrapolated to humans. As another possibility, in vitro studies report that PFASs may bind to phospholipid membranes, thereby influencing membrane fluidity [38]. Thus, given the long half-life of the PFASs in human blood [1], accumulation in membranes might well cause long-term adverse effects on cell functions including lipid metabolism. Of note, we cannot exclude the possibility that our observed associations may not bear any causal interpretation if PFASs are incorporated in the same lipoprotein species that contain apoC-III, although we believe that such possibility is small because the current evidence suggests that the majority of PFASs in circulation are carried by albumin rather than lipoproteins [39]. In addition, in a sensitivity analysis, we observed similar results when we examined the ratio of IDL, LDL, and HDL particles that contain apoC-III to total cholesterol levels as a surrogate measure of lipoprotein compositions. More studies are warranted to elucidate the distribution of PFASs in blood compartments and other tissues in human body.

To our knowledge, this is among the first studies to investigate the associations between PFAS exposure and lipoprotein and apolipoprotein subspecies in adults. The present study accounted for a multitude of potential confounding factors, including diet and lifestyle factors, BMI, and lipid-lowering medication use. However, our study is also subject to some limitations. First, our primary findings were based on a cross-sectional analysis, although similar patterns of associations were observed between baseline PFAS concentrations and lipoprotein and apolipoproteins measured 2 years later. Second, our study participants were overweight or obese and had relatively homogeneous health status, and therefore our findings may not be extrapolated to populations with other characteristics. Third, the role of multiple testing must be considered, although we chose not to apply the conservative Bonferroni correction in the analyses given the inter-correlation between the PFASs $\left(r_{\mathrm{s}}\right.$ ranged from 0.4 to 0.9 ). Fourth, we measured only baseline plasma PFAS concentrations and could not examine the associations of changes in PFASs and lipoprotein subspecies during the follow-up. Fifth, the associations tended to be more pronounced in women, but we do not have sufficient statistical power to formally test potential gender or racial differences. Finally, residual or unmeasured confounding could not be entirely ruled out in this observational study.

\section{Conclusions}

Plasma PFAS levels were not associated with blood lipids in overweight or obese individuals who participated in a clinical trial, although plasma levels of PFOA were significantly associated with higher apoC-III, as well as levels of cholesterol, triglycerides, and other apolipoproteins in subspecies of IDL, LDL, and HDL that contain apoC-III. These novel findings suggest that PFAS exposures might interfere with lipid metabolism not reflected by routine lipid parameters and that the potential detrimental effects of PFASs on promoting atherogenic lipid subfractions may potentially lead to an elevated risk of developing CVD.

\section{Supplementary information}

Supplementary information accompanies this paper at https://doi.org/10. 1186/s12940-020-0561-8.

\footnotetext{
Additional file 1: Figure S1. Heatmap of correlations between lipoprotein and apolipoprotein subspecies*. Table S1. Comparisons of characteristics between included and excluded participants. Table S2. Partial Spearman correlation coefficients between baseline PFASs and lipids and apolipoproteins in VLDL. Table S3. Partial Spearman correlation coefficients between PFASs and lipoproteins and apolipoproteins in men and women. Table S4. Partial Spearman correlation coefficients between baseline PFASs and lipoproteins and apolipoproteins at 24th month after the diet intervention.
} 


\section{Authors' contributions}

$\mathrm{GL}$ and QS supervised the research project; GL analyzed and interpreted the data; $G L$ wrote the first draft of the manuscript; $B Z, Y H, J R, L L, L Q, G B, L D, P G$, $J F$, QS revised and provided comments to the manuscript. All authors read and approved the final manuscript.

\section{Funding}

This research was supported by $\mathrm{NIH}$ grants from the National Institute for Environmental Health Sciences (ES022981, ES021372, ES021477, and ES027706), the National Heart, Lung, and Blood Institute (HL073286), and the General Clinical Research Center, National Institutes of Health (RR-02635). The funders had no role in study design, data collection and analysis, decision to publish, or preparation of the manuscript. The funding source had no involvement in study design; in the collection, analysis and interpretation of data; in the writing of the report; and in the decision to submit the article for publication

\section{Availability of data and materials}

The data supporting the conclusions of this work are included within the manuscript and its Additional files. Further, the dataset analyzed during the current study is available from the corresponding author on reasonable request.

\section{Ethics approval and consent to participate}

The protocol was approved by the Institutional Review Board at Harvard T.H. Chan School of Public Health, Brigham and Women's Hospital, and the Pennington Biomedical Research Center of the Louisiana State University System, as well as by a data and safety monitoring board appointed by the National Heart, Lung, and Blood Institute. All participants provided written informed consent.

\section{Consent for publication}

Not applicable.

\section{Competing interests}

The authors declare that they have no competing interests associated with this manuscript. PG served as a health expert for the State of Minnesota in a lawsuit against a PFAS-producing company.

\section{Author details}

'Department of Nutrition and Food Hygiene, Hubei Key Laboratory of Food Nutrition and Safety, Ministry of Education Key Laboratory of Environment and Health, School of Public Health, Tongji Medical College, Huazhong University of Science and Technology, Wuhan, China. ${ }^{2}$ Department of Nutrition, Harvard T.H. Chan School of Public Health, 665 Huntington Ave, Boston, MA 02115, USA. ${ }^{3}$ Department of Biochemistry, Fukuoka University School of Medicine, Fukuoka, Japan. ${ }^{4}$ Pennington Biomedical Research Center, LSU, Baton Rouge, LA, USA. ${ }^{5}$ Department of Epidemiology and Department of Biostatistics, Harvard T.H. Chan School of Public Health, Boston, MA, USA. ${ }^{6}$ Department of Epidemiology, School of Public Health and Tropical Medicine, Tulane University, New Orleans, LA, USA. ${ }^{7}$ Department of Nutrition and Food Studies, George Mason University, Fairfax, VA, USA. ${ }^{8}$ Department of Biostatistics, Harvard T.H. Chan School of Public Health, Boston, MA, USA. IInstitute of Public Health, University of Southern Denmark, Odense, Denmark. ${ }^{10}$ Department of Envrionmental Health, Harvard T.H. Chan School of Public Health, Boston, MA, USA. ${ }^{11}$ Channing Division of Network Medicine, Department of Medicine, Brigham and Women's Hospital and Harvard Medical School, Boston, MA, USA.

\section{Received: 4 July 2019 Accepted: 3 January 2020}

\section{Published online: 13 January 2020}

\section{References}

1. Agency for Toxic Substances and Disease Registry. Draft toxicological profile for perfluoroalkyls. Atlanta: Agency for Toxic Substances and Disease Registry; 2018.

2. Fletcher T, Galloway TS, Melzer D, Holcroft P, Cipelli R, Pilling LC, Mondal D, Luster M, Harries LW. Associations between PFOA, PFOS and changes in the expression of genes involved in cholesterol metabolism in humans. Environ Int. 2013;57-58:2-10.

3. Wolf CJ, Schmid JE, Lau C, Abbott BD. Activation of mouse and human peroxisome proliferator-activated receptor-alpha (PPARalpha) by perfluoroalkyl acids (PFAAs): further investigation of C4-C12 compounds. Reprod Toxicol. 2012;33(4):546-51.

4. Geiger SD, Xiao J, Ducatman A, Frisbee S, Innes K, Shankar A. The association between PFOA, PFOS and serum lipid levels in adolescents. Chemosphere. 2014;98:78-83.

5. Nelson JW, Hatch EE, Webster TF. Exposure to polyfluoroalkyl chemicals and cholesterol, body weight, and insulin resistance in the general U.S. population. Environ Health Perspect. 2010;118(2):197-202.

6. Steenland K, Tinker S, Frisbee S, Ducatman A, Vaccarino V. Association of perfluorooctanoic acid and perfluorooctane sulfonate with serum lipids among adults living near a chemical plant. Am J Epidemiol. 2009;170(10): 1268-78.

7. Frisbee SJ, Shankar A, Knox SS, Steenland K, Savitz DA, Fletcher T, Ducatman AM. Perfluorooctanoic acid, perfluorooctanesulfonate, and serum lipids in children and adolescents: results from the $\mathrm{C} 8$ health project. Arch Pediatr Adolesc Med. 2010;164(9):860-9.

8. Fitz-Simon N, Fletcher T, Luster MI, Steenland K, Calafat AM, Kato K, Armstrong B. Reductions in serum lipids with a 4-year decline in serum perfluorooctanoic acid and perfluorooctanesulfonic acid. Epidemiology. 2013;24(4):569-76

9. Winquist A, Steenland K. Modeled PFOA exposure and coronary artery disease, hypertension, and high cholesterol in community and worker cohorts. Environ Health Perspect. 2014;122(12):1299-305.

10. Fisher M, Arbuckle TE, Wade M, Haines DA. Do perfluoroalkyl substances affect metabolic function and plasma lipids?--Analysis of the 2007-2009, Canadian health measures survey (CHMS) cycle 1. Environ Res. 2013;121:95-103.

11. Donat-Vargas C, Bergdahl IA, Tornevi A, Wennberg M, Sommar J, Koponen J, Kiviranta $\mathrm{H}$, Akesson A. Associations between repeated measure of plasma perfluoroalkyl substances and cardiometabolic risk factors. Environ Int. 2019; 124:58-65.

12. Grandjean P. Health status of workers exposed to perfluorinated alkylate substances (letter). J Occup Environ Med. 2018;60:e562 (in press).

13. Feingold KR, Grunfeld C. Introduction to lipids and lipoproteins; 2000.

14. Krauss RM. Lipoprotein subfractions and cardiovascular disease risk. Curr Opin Lipidol. 2010;21(4):305-11.

15. Alaupovic P. Significance of apolipoproteins for structure, function, and classification of plasma lipoproteins. Methods Enzymol. 1996;263:32-60.

16. Jensen MK, Rimm EB, Furtado JD, Sacks FM. Apolipoprotein C-III as a Potential Modulator of the Association Between HDL-Cholesterol and Incident Coronary Heart Disease. J Am Heart Assoc. 2012;1:jah3-e000232.

17. Morton AM, Koch M, Mendivil CO, Furtado JD, Tjonneland A, Overvad K, Wang $L$, Jensen MK, Sacks FM. Apolipoproteins E and CIII interact to regulate HDL metabolism and coronary heart disease risk. JCl Insight. 2018;3(4):e98045.

18. Mendivil CO, Rimm EB, Furtado J, Chiuve SE, Sacks FM. Low-density lipoproteins containing apolipoprotein C-III and the risk of coronary heart disease. Circulation. 2011;124(19):2065-72.

19. Kohan AB. Apolipoprotein C-III: a potent modulator of hypertriglyceridemia and cardiovascular disease. Curr Opin Endocrinol Diabetes Obes. 2015;22(2):119-25.

20. Wyler von Ballmoos MC, Haring B, Sacks FM. The risk of cardiovascular events with increased apolipoprotein CIII: A systematic review and metaanalysis. J Clin Lipidol. 2015;9(4):498-510.

21. Sacks FM, Bray GA, Carey VJ, Smith SR, Ryan DH, Anton SD, McManus K, Champagne CM, Bishop LM, Laranjo N, et al. Comparison of weight-loss diets with different compositions of fat, protein, and carbohydrates. N Engl J Med. 2009;360(9):859-73.

22. Haug LS, Thomsen C, Becher G. A sensitive method for determination of a broad range of perfluorinated compounds in serum suitable for large-scale human biomonitoring. J Chromatogr A. 2009;1216(3):385-93.

23. Calafat AM, Wong LY, Kuklenyik Z, Reidy JA, Needham LL. Polyfluoroalkyl chemicals in the U.S. population: data from the National Health and nutrition examination survey (NHANES) 2003-2004 and comparisons with NHANES 1999-2000. Environ Health Perspect. 2007;115(11):1596-602.

24. Furtado JD, Campos H, Appel LJ, Miller ER, Laranjo N, Carey VJ, Sacks FM. Effect of protein, unsaturated fat, and carbohydrate intakes on plasma apolipoprotein B and VLDL and LDL containing apolipoprotein C-III: results from the OmniHeart trial. Am J Clin Nutr. 2008;87(6):1623-30.

25. Liu G, Dhana K, Furtado JD, Rood J, Zong G, Liang L, Qi L, Bray GA, DeJonge $L$, Coull B, et al. Perfluoroalkyl substances and changes in body weight and resting metabolic rate in response to weight-loss diets: a prospective study. PLoS Med. 2018;15(2):e1002502. 
26. Baecke JA, Burema J, Frijters JE. A short questionnaire for the measurement of habitual physical activity in epidemiological studies. Am J Clin Nutr. 1982; 36(5):936-42.

27. Steenland K, Fletcher T, Savitz DA. Epidemiologic evidence on the health effects of perfluorooctanoic acid (PFOA). Environ Health Perspect. 2010; 118(8):1100-8.

28. Lin CY, Wen LL, Lin LY, Wen TW, Lien GW, Chen CY, Hsu SH, Chien KL, Sung $F C$, Chen PC, et al. Associations between levels of serum perfluorinated chemicals and adiponectin in a young hypertension cohort in Taiwan. Environ Sci Technol. 2011;45(24):10691-8.

29. Sunderland EM, Hu XC, Dassuncao C, Tokranov AK, Wagner CC, Allen JG. A review of the pathways of human exposure to poly-and perfluoroalkyl substances (PFASs) and present understanding of health effects. J Expo Sci Environ Epidemiol. 2019;29(2):131-47.

30. Zeng XW, Qian Z, Emo B, Vaughn M, Bao J, Qin XD, Zhu Y, Li J, Lee YL, Dong GH. Association of polyfluoroalkyl chemical exposure with serum lipids in children. Sci Total Environ. 2015;512-513:364-70.

31. Starling AP, Engel SM, Whitworth KW, Richardson DB, Stuebe AM, Daniels JL, Haug LS, Eggesbo M, Becher G, Sabaredzovic A, et al. Perfluoroalkyl substances and lipid concentrations in plasma during pregnancy among women in the Norwegian mother and child cohort study. Environ Int. 2014; 62:104-12.

32. Chateau-Degat ML, Pereg D, Dallaire R, Ayotte P, Dery S, Dewailly E. Effects of perfluorooctanesulfonate exposure on plasma lipid levels in the Inuit population of Nunavik (northern Quebec). Environ Res. 2010;110(7):710-7.

33. Sacks FM, Alaupovic P, Moye LA, Cole TG, Sussex B, Stampfer MJ, Pfeffer MA, Braunwald E. VLDL, apolipoproteins B, CIII, and E, and risk of recurrent coronary events in the cholesterol and recurrent events (CARE) trial. Circulation. 2000;102(16):1886-92.

34. Talayero B, Wang L, Furtado J, Carey VJ, Bray GA, Sacks FM. Obesity favors apolipoprotein E- and C-III-containing high density lipoprotein subfractions associated with risk of heart disease. J Lipid Res. 2014;55(10):2167-77.

35. Huang M, Jiao J, Zhuang P, Chen X, Wang J, Zhang Y. Serum polyfluoroalkyl chemicals are associated with risk of cardiovascular diseases in national US population. Environ Int. 2018;119:37-46.

36. Scharmach E, Buhrke T, Lichtenstein D, Lampen A. Perfluorooctanoic acid affects the activity of the hepatocyte nuclear factor 4 alpha (HNF4alpha). Toxicol Lett. 2012;212(2):106-12.

37. Hayhurst GP, Lee YH, Lambert G, Ward JM, Gonzalez FJ. Hepatocyte nuclear factor $4 a$ lpha (nuclear receptor 2A1) is essential for maintenance of hepatic gene expression and lipid homeostasis. Mol Cell Biol. 2001;21(4):1393-403.

38. Sanchez Garcia D, Sjodin M, Hellstrandh M, Norinder U, Nikiforova V, Lindberg J, Wincent E, Bergman A, Cotgreave I, Munic Kos V. Cellular accumulation and lipid binding of perfluorinated alkylated substances (PFASs) - a comparison with lysosomotropic drugs. Chem Biol Interact. 2018:281:1-10.

39. Zhang $X$, Chen L, Fei XC, Ma YS, Gao HW. Binding of PFOS to serum albumin and DNA: insight into the molecular toxicity of perfluorochemicals. BMC Mol Biol. 2009;10:16.

\section{Publisher's Note}

Springer Nature remains neutral with regard to jurisdictional claims in published maps and institutional affiliations.

Ready to submit your research? Choose BMC and benefit from:

- fast, convenient online submission

- thorough peer review by experienced researchers in your field

- rapid publication on acceptance

- support for research data, including large and complex data types

- gold Open Access which fosters wider collaboration and increased citations

- maximum visibility for your research: over $100 \mathrm{M}$ website views per year

At BMC, research is always in progress.

Learn more biomedcentral.com/submissions 